\section{Operant behavior as an optimally controlled process*}

\author{
MICHAEL F. HALASZ \\ University of Manitoba, Winnipeg, Manitoba, Canada
}

The concept of conditioned behavior as servomechanistically driven and homeostatically stabilized goes back to W. Ross Ashby's classical book, Design for a brain (1960). The idea has been given concrete realization in operant settings (Staddon, 1964; Halasz, 1966, 1968, 1969,1970 ) with attendant literature providing theoretical explanation. We now have generalized it in accordance with a more modern diagram of optimally controlled processes (Merriam, 1964).

The process control diagram consists of the following blocks:

(1) The controlled process. This includes not only the $S$ in the chamber, but all static (fixed) feedback loops provided not only by proprioceptive circuits under Ss' skin, but by the reinforcement schedule programming apparatus maintaining contingencies.

(2) The performance measure system. This block receives output information from the controlled process block and information from the demand generator (representing the performance requirements of the $E$ or of automated equipment). The comparison of actual output behavior with the demand level yields the error function for each conditioning trial or subperiod. The error function is then integrated, smoothed, corrected for stochastic fluctuations, etc., to form the error index, which is the basis for the decisions of the statistical inference and decision system.

(3) The statistical inference and decision system. This block performs the computations on the error index necessary to make decisions about the application or removal of the control signal.

(4) The control signal. This signal is felt by the controlled process block whenever the process requires "correction." In optimal control, signal has an "all-or-none" character applied in "bang-bang" fashion, i.e., switched in and out in such a way as to make the correctional action most rapid and stable. When the process

\footnotetext{
* Version of a paper presented to National Conference on the Use of On-Line Computers in Psychology, St. Louis, Missouri, November 10, 1971.
}

control is exerted over an operant, the control signal consists of a change of reinforcement schedule, achieved by a correctional alteration within the feedback loop leading from effector activity to the reception of reinforcing stimuli via the programming apparatus. Optimal control of operant behavior is best understood by representing it in a phase plane. The coordinates are the error index $P$ and its time derivative $\mathrm{dP} / \mathrm{dT}$. Thus, the phase plane not only represents the level of the controlled behavior, but also its tendencies to increase or decrease that level. The origin, corresponding to the demanded or best level of response with no tendency to leave it, constitutes the equilibrium point about which the behavior is to be regulated. If the equilibrium point is unstable, the trajectory representing the history of behavior will start to diverge from the origin. The optimal control arrangement returns it there as quickly as possible and with minimum oscillation. To this end, the correctional control signal (reinforcement schedule change) is applied when the trajectory crosses a certain switching line in the phase plane. A vertical switching line represents application of the control signal when the behavioral error index reaches a certain tolerance level. An oblique line indicates attention to tendencies in behavior (anticipatory derivative control) in making the decision to switch in the correctional schedule change. The slide shows such an oblique switching line reversing the trend toward excessively high response levels and resulting in a spiral reconvergence of the trajectory about the equilibrium point. This case corresponds to divergence from an unstable node, followed by a damped oscillation back toward a stable focus (Minorsky, 1962). The switching lines are simple cases of the application of various control policies. If the system equations are known, the optimal control policy may be derived mathematically. In the behavioral case, we are, of course, confronted with the inverse problem: we empirically probe the behavioral system with various control policies; determining the optimal one (from the point of view of rapidity and stability of the resultant adjustment) gives insight into the unknown governing equation.

The on-line computing capability required in our block diagram pertains to the performance measure and statistical decision elements of the system. Necessary mathematical operations are averaging of performance data over trial and time intervals, differencing, integrating, and estimating of derivatives from finite data differences. In the absence of on-line digital computer capability, we accomplish these analogically. Heathkit chopper-stabilized operational amplifiers are used for integrations, subtractions, etc. In steps where multiplication must be performed, Motorola 4-Quadrant Multipliers are used; these are themselves operational amplifiers, with the feedback impedance dependent on one of the multiplicands. The 4-Quadrant Multipliers are essential in the control of the variance of responsemeasure distributions or, in general, of higher moments than the mean performance measure. The analog computing system interfaces with regular BRS programming equipment (fixed limb of the feedback diagram) by way of Schmitt triggers and Rovetti "windows." As the computed error index exceeds threshold levels, the logic activates relays that alter the values of timing capacitors in the programming apparatus. Analog systems are very inexpensive and convenient but require much calibration and general electronic sophistication in those who operate them.

As developed in our laboratory, the concept of operant behavior as an optimally controlled process has the following applications: (1) automated "sh a ping" procedure, (2) sensitive "probe" methodology for exploring behavioral abnormality in medicated or brain-lesioned animals, (3) teaching of control concepts to psychology students, and (4) simulation of "higher" CNS control over habits complete at "lower" brain levels.

We view the fusion of cybernetic with behavioral terminology as very promising for psychology.

\section{REFERENCES}

ASHBY, W. R. Design for a brain. London: Wiley, 1960 .

HALASZ, M. F. Stability of conditioned delay behavior computed from transient response to perturbation of reinforcement schedule. American Zoologist, 1966, 6, 543.

HALASZ, M. F. A behavioural evoked response: Probing the stability of delayed conditioned approach with impulse-like changes of reinforcement schedule. Canadian Journal of Psychology, 1968 , 22, 229-243.

HALASZ, M. F, \& CHENG, Y. Modulation of delayed conditioned approach with correctional changes of reinforcement schedule: Integral/optimal control. $77^{\text {th }}$ 
American Psychological Association Proceedings, 1969, 4, 833-834.

HALASZ, M. F. Transitions of conditioned delay in a behavioral phase-plane: Nodes, foci and limit cycles. International Journal of Psychobiology, 1970, 1, 43-47.

\section{Measuring cooperation and competition}

\section{in decomposed games}

\section{JAMES W. LIVINGSTON, JR. \\ Rutgers University, New Brunswick, New Jersey 08903}

The topic of my presentation is not a new development in either hardware or software; rather, it is an application of some of the power provided by an interactive time-sharing system-the University of California at Santa Barbara On-Line System (UCSB OLS), a variant of the Culler-Fried On-Line System (see, e.g., Sammett, 1969, for a description). UCSB OLS is of great interest, I think, for at least three reasons apart from its power. First, OLS is available to prospective users via direct export of the software which supports it. Second, OLS may be written for a small computer; a version of it has been implemented on a 16-bit minicomputer at Santa Barbara (SEL-810B). Third, one may obtain access to OLS via a dial-up terminal, which may be obtained from UCSB. Finally, UCSB OLS is a terminal-oriented system; it uses a double keyboard/storage CRT terminal (Tektronix 601 or 611 storage display oscilloscope), thus providing graphics capability at much lower cost than many other similar terminals. To set the context for the application, a few psychologically substantive introductory remarks are in order.

Matrix games, in general, and the PD game, in particular, have been used as prototypes of interdependent choice situations by a number of investigators (see, e.g., Becker \& McClintock, 1967, and Vinacke, 1969, for reviews of that literature). One may "decompose" the usual game matrix in such a way as to make less salient the contribution of the player's counterpart (Pruitt, 1967; Messick \& McClintock, 1968). The picture presented by the decomposed game (DG) matrix is suggestive of an analysis scheme which may help explicate the player's behavior in these situations. That is, if the player sees the display
MERRIAM. Optimization theory and the design of feedback systems. New York: McGraw-Hill, 1964.

MINORSKY, N. Nonlinear oscillations. New York: Van Nostrand, 1962

STADDON, J. E. R. Reinforcement as Science, $1961,145,410-412$. input: Cyclic variable-interval schedule.

UCSB OLS supports. It does so for an individual $S$, who is seated in a small experimental room with the CRT and a four-key subset of the OLS keyboard.

The experimental arrangement provides for $\mathrm{E}$ control of the entire experiment, since the $E$ has the full keyboard, as well as a view of the CRT through a one-way window. Various arrangements of the situation are possible. Two Ss can be run in different rooms, playing each other, each playing a model, one engaged with no "other" at all, and so forth. The CRT output makes it simple to manipulate the game display or feedback on "other's" choices; these two factors are often of importance as independent variables.

There are a few disadvantages to the arrangement I've described. First, there are only a limited number of OLS ports available at UCSB, so access via dial-up terminal may not be available for too long. Second, like any timed-shared, computer-managed experimental apparatus, the operation of this one is subject to the vagaries of the particular computer center supporting it. While service has been good for at least 2 years, it was not always so. This last difficulty seems less important than some which attend the acquisition of an arrangement like the one described, but it can make a difference in the business of doing research.

Finally, I must note that research has been done with the apparatus. Three experiments, one of which was a Monte-Carlo study of a stochastic model (Messick \& McClintock, 1968) of choice behavior, were done. Results were pretty much in accord with expectations. Ss will choose in some consistently classifiable way, by and large, and the manipulation of some reasonable variables, e.g., the relative advantage of the player or his counterpart, makes a difference in the position of the $S$ along the competition-cooperation continuum. Moreover, the estimation procedure does not seem to discriminate the nature of the choice mechanism it is measuring. It is quite able to output a value of $\mu$ for most possible choice processes. That result is particularly interesting, since the estimation procedure was generated because of a need to estimate the parameter of a model for the choice process. What we have finally, however, is a general technique for summarizing choices in the DG.

mes to a player in such a that each pair gets closer together and both approach the direction indicated by the player's choice, one gets convergence to a pair of alternatives which brackets $\mu$ as closely as desired. It is exactly the iteration of this convergence procedure that

\section{REFERENCES} Value: Behavioral decision theory. Annual Review of Psychology, 1967, 18, 239-286.

MESSICK, D. M., \& McCLINTOCK, C. G. Motivational bases of choice in
BECKER, G. M., \& MCCLINTOCK, C. G. 